\title{
Probabilistic methods in BCI research
}

\author{
P. Sykacek, S. Roberts, M. Stokes, E. Curran, M. Gibbs and L. Pickup
}

\begin{abstract}
This paper suggests a probabilistic treatment of the signal processing part of a brain computer interface (BCI). We suggest two improvements for BCIs that can-not be obtained easily with other data driven approaches. Simply by using one large joint distribution as a model of the entire signal processing part of the BCI, we can obtain predictions that implicitly weight information according to its certainty. Offline experiments reveal that this results in statistically significant higher bit rates. Probabilistic methods are also very useful to obtain adaptive learning algorithms that can cope with non-stationary problems. An experimental evaluation shows that an adaptive BCI outperforms the equivalent static implementations, even when using only a moderate number of trials. This suggests that adaptive translation algorithms might help in cases where brain dynamics change due to learning effects or fatigue.
\end{abstract}

\section{INTRODUCTION}

The BCI used in our paper follows previous work by [3], [14], [8] and [7] and uses surface electroencephalography (EEG) to record brain signals that are converted into computer commands. A good average communication bandwidth of such a system will be in the range of 5-25 bit per minute [13]. This is sufficient for non time critical communication tasks like [3], but clearly not enough for high bandwidth applications such as control of a powered wheel chair. A probabilistic point of view of current BCI signal processing suggests two modifications that have been found to improve the bandwidth. Probabilistic methods (e.g. [2]) regard all BCI parameters (i.e. features extracted from EEG and all other parameters) as random variables. Inference (i.e. finding the posterior distribution $p(\boldsymbol{w} \mid \mathcal{D})$ over model parameters $\boldsymbol{w}$ given data $\left.\mathcal{D}=\left\{y_{n} \forall n=1 . . N\right\}\right)$ and prediction, require two operations.

- Bayes' theorem $p(\boldsymbol{w} \mid \mathcal{D})=p(\boldsymbol{w}) p(\mathcal{D} \mid \boldsymbol{w}) / p(\mathcal{D})$ converts a prior, $p(\boldsymbol{w})$, and a likelihood, $p(\mathcal{D} \mid \boldsymbol{w})$ into a posterior.

- To obtain model predictions $\hat{y}=\int_{\boldsymbol{w}} y(\boldsymbol{w}) p(\boldsymbol{w} \mid \mathcal{D}) d \boldsymbol{w}$ and the normalization constant $p(\mathcal{D})=\int_{\boldsymbol{w}} p(\boldsymbol{w}) p(\mathcal{D} \mid \boldsymbol{w}) d \boldsymbol{w}$ we need to calculate marginalization integrals.

We use probabilistic methods to improve two suboptimal aspects of current BCI systems. First we compare classical time series classification with a probabilistic generalization that uses a joint model for feature extraction and classification. Used in that manner, the probabilistic approach implicitly considers the certainty of information. Empirical tests confirm that this allows us to predict cognitive states with higher accuracy and thus to increase the communication bandwidth. The second aspect of probabilistic methods considered in this paper is to use a hierarchical model

P. Sykacek, S. Roberts, M. Gibbs and L. Pickup work in the Department of Engineering Science at the University of Oxford, Parks Road, OX1 3PJ Oxford, UK, (E-mails: [psyk,sjrob,mgibbs,elle]@robots.ox.ac.uk). M. Stokes works in the Research Department of the Royal Hospital for Neuro-disability, West Hill, SW15 3SW London. E. Curran works in the School and Department of Law, Keele University, ST5 5BG Keele, UK for infering the BCI classifier adaptively. Compared with a stationary model, the adaptive BCI shows a statistically significant improvement in communication bandwidth.

\section{Methods}

\section{A. Data collection}

The BCI data used in this paper for retrospective studies was obtained from 10 young, healthy and untrained volunteers, who underwent two different types of experiments. Each experiment consisted of 10 trials of 7 seconds duration. The timing of the experiment was controlled by visual indication on the computer screen allowing for a period of rest between trials. For the first experiment, in tables I and II refered to as "aud./navig.", the volunteers were asked to alternate to imagine singing a familiar song and to imagine walking around in a familiar environment. During these trials the subjects EEG was recorded at two electrode positions: T4 against P4 and C3' against C3", where $\mathrm{C} 3$ ' and $\mathrm{C} 3$ " are $3 \mathrm{~cm}$ anterior and posterior to the standard position C3. In the second experiment, in the tables I and II refered to as "left/right", the subjects were asked to alternate between imagined left and right hand movements. This time EEG was recorded at positions C3' against C3" and positions C4' against C4", the latter being $3 \mathrm{~cm}$ anterior and posterior to position $\mathrm{C} 4$. In both experiments the ground electrode was placed at position A1. Data were recorded with an ISO-DAM system using a gain of $10^{4}$ and a fourth order band pass filter with pass band between $0.1 \mathrm{~Hz}$ and $100 \mathrm{~Hz}$. The EEG signals were sampled with $384 \mathrm{~Hz}$ and 12 bit resolution. The EEG of all trials was manually checked for artefacts such as EOG and EMG contamination. If such artefacts were found, the trial was repeated. Our experiments use these signals without further filtering.

\section{B. Performance comparisons}

A data driven approach to BCI research requires evaluating different methods. As is pointed out in [13] the performance of a BCI should be quantified by the bit rate obtained on previously unseen data. Leaving the rate of prediction constant, this bit rate is proportional to the generalization accuracy of the BCI classifier which estimates the proportion of correct classifications we may expect when using the system for previously unseen BCI experiments. This connection readily leads to means for assessing the statistical significance of observed differences. The appropriate method to compare two BCI systems is to use $\mathrm{McNe}$ mar's test (e.g. [9], page 77). Differences in classification accuracy are only determined by those samples in the test data that were labeled differently. The idea of McNemar's test is to count the number of errors made by the first clas- 
sifier that are correctly classified by the second, $n_{a}$, and vice versa to count all mistakes of the second classifier that were not made by the first, $n_{b}$. The null hypothesis that both classifiers are equal is then given by the binomial distribution $\mathcal{B}_{n}\left(0.5,\left(n_{a}+n_{b}\right)\right.$ ). Assuming that $n_{b}>n_{a}$ (otherwise we exchange $n_{a}$ and $\left.n_{b}\right)$ and using $P_{\mathcal{B}_{n}}\left(i, n_{b}+i\right)$ as probability of observing $\left(i, n_{b}+i\right)$ under the null hypothesis, we reject the null hypothesis if the probability $P_{\text {null }}=\sum_{i=1}^{n_{a}} P_{\mathcal{B}_{n}}\left(i, n_{b}+i\right)$ is below 0.05 .

\section{Fully probabilistic BCI}

A standard implementation of the signal processing part of a BCI separates into feature extraction, which might for example use an autoregressive (AR) model, and classification. Probabilistic methods have with success been used for signal processing applications (e.g. [10]) and for classification (e.g. [5]). As a consequence of probabilistic principles, separating signal processing and classification must be regarded as approximation. We thus suggest to use the implementation of probabilistic time series classification in [11] as fully probabilistic BCI. Unlike modeling signal processing and classification separately, we use one joint density over all involved model parameters. Predicting the probability of a cognitive state for an EEG segment requires marginalizing over all unknown quantities. In order to illustrate the idea of the algorithm, let us assume that we want to predict the probability of cognitive state $y$ from two EEG segments, $\mathcal{X}_{a}$ and $\mathcal{X}_{b}$, that were recorded simultaneously at two different electrodes. We further assume that we model both segments by AR models with coefficients $\varphi_{a}$ and $\varphi_{b}$ and model orders $I_{a}$ and $I_{b}$. We obtain $P\left(y \mid \mathcal{X}_{a}, \mathcal{X}_{b}\right)$ by integrating over $\varphi_{a}, I_{a}, \varphi_{b}$ and $I_{b}$. Assuming conditional independence of the EEG channels given cognitive state $y$, we may express this as

$$
\begin{aligned}
& P\left(y \mid \mathcal{X}_{a}, \mathcal{X}_{b}\right)=\frac{p\left(\mathcal{X}_{a}\right) p\left(\mathcal{X}_{b}\right)}{p\left(\mathcal{X}_{a}, \mathcal{X}_{b}\right)} \frac{1}{P(y)} \\
& \quad \times \quad\left(\sum_{I_{a}} \int_{\boldsymbol{\varphi}_{a}=-\infty}^{\infty} P\left(y \mid \boldsymbol{\varphi}_{a}, I_{a}\right) p\left(\boldsymbol{\varphi}_{a}, I_{a} \mid \mathcal{X}_{a}\right) d \boldsymbol{\varphi}_{a}\right. \\
& \left.\quad \times \quad \sum_{I_{b}} \int_{\boldsymbol{\varphi}_{b}=-\infty}^{\infty} P\left(y \mid \boldsymbol{\varphi}_{b}, I_{b}\right) p\left(\boldsymbol{\varphi}_{b}, I_{b} \mid \mathcal{X}_{b}\right) d \boldsymbol{\varphi}_{b}\right),
\end{aligned}
$$

where $P(y)$ denotes prior probability and $\frac{p\left(\mathcal{X}_{a}\right) p\left(\mathcal{X}_{b}\right)}{p\left(\mathcal{X}_{a}, \mathcal{X}_{b}\right)}$ is a normalizing constant.Since a BCI operates on a temporal sequence of EEG segments, subsequent predictions will be correlated. Hidden Markov models (HMM), which have been applied to BCI in [6], can model these correlations. Unlike the approach in [6], we just want to allow for information flow across time and thus use only one HMM. We model one second segments for every EEG electrode separately with AR models allowing for model orders between 0 and 5. Inference requires computing integrals similar to Equation (1), which are analytically intractable. We thus use numerical integration based on Markov chain Monte Carlo methods (see [11] for details).

Table I shows the average within subject results we obtain with this paradigm. Evaluation is done by 10 fold cross
TABLE I

COMmUNiCATION BANDWIDTh OF A FULLY PROBABILISTIC METHOD VS. CLASSICAL TIME SERIES CLASSIFICATION

\begin{tabular}{|c|c|c|c|}
\hline cognitive tasks & classical & full Bayes & $P_{\text {null }}$ \\
\hline aud. / navig. & $0.21 \mathrm{bit} / \mathrm{s}$ & $0.38 \mathrm{bit} / \mathrm{s}$ & $\ll 0.01$ \\
\hline left / right & $0.20 \mathrm{bit} / \mathrm{s}$ & $0.31 \mathrm{bit} / \mathrm{s}$ & $\ll 0.01$ \\
\hline
\end{tabular}

validation using in every fold 14 samples as test data and 126 samples as training data. We compare the communication bandwidth obtained with the fully probabilistic approach (column full Bayes) with results obtained using the same classical approach with signal processing and classification done separately (column classical). The probabilities of the null hypothesis of McNemar's test in column $P_{\text {null }}$ show that the difference between a fully probabilistic model and a classical approach is statistically significant. Despite having found that a fully Bayesian approach improves BCI performance, the proposed method can for computational reasons not be directly applied to online BCI. This suggests looking for approximations which can be used in real time and nevertheless achieve the desired effects.

\section{A probabilistic implementation of adaptive BCI}

Signal processing algorithms as used in current BCIs assume stationarity. This is in contrast to [7], who suggest that relations between EEG dynamics and cognitive states are not stationary. Such non-stationarities can have technical reasons (e.g. changing electrode impedance). However they might also be caused by learning effects or fatigue of the subject. Assuming that EEG dynamics are represented by AR coefficients $\varphi_{n}$, we can describe the problem as a non stationary joint distribution $p\left(y_{n}, \boldsymbol{\varphi}_{n}\right)_{n}$, over AR coefficients and cognitive task label $y_{n}$. Time dependency is indicated by index $n$. In order to obtain a real time approach, we suggest using a generalized linear classifier parameterized by time varying coefficients $\boldsymbol{w}_{n}$. A probabilistic graph that illustrates the suggested approach is shown in Fig. 1. We assume a model that predicts the probabilities of cognitive states $y_{n}$ and regard the parameters of the classifier as state variables in a first order Markov process. Inference of the adaptive classifier is derived as transition from $\boldsymbol{w}_{n-1}$ to $\boldsymbol{w}_{n}$. Using isotropic zero mean Gaussian process noise, i.e. $p\left(\boldsymbol{w}_{n}, \mid \boldsymbol{w}_{n-1}, \lambda\right)=\mathcal{N}\left(\boldsymbol{w}_{n-1} ; \lambda \boldsymbol{I}\right)$ with inverse covariance matrix $\lambda \boldsymbol{I}$, we obtain the posterior at time $n$ as

$$
\begin{aligned}
& p\left(\boldsymbol{w}_{n} \mid y_{n}, \mathcal{D}_{n-1}\right)=\int_{\boldsymbol{w}_{n-1}} \int_{\lambda} \frac{p\left(y_{n} \mid \boldsymbol{w}_{n}\right)}{p\left(y_{n}\right)} \\
& \quad \times \quad p\left(\boldsymbol{w}_{n}, \mid \boldsymbol{w}_{n-1}, \lambda\right) p\left(\boldsymbol{w}_{n-1} \mid, \mathcal{D}_{n-1}\right) p(\lambda) d \boldsymbol{w}_{n-1} d \lambda,
\end{aligned}
$$

where $p\left(\boldsymbol{w}_{n-1} \mid, \mathcal{D}_{n-1}\right)$ denotes the posterior at time $n-1$ and $p\left(y_{n}\right)$ is a normalization constant. The advantage of a probabilistic implementation over other approaches is that hierarchically infering $\lambda$ from a window of appropriate size, actually determines the appropriate "learning rate". This is much more difficult with other approaches. In a linear Gaussian case inference according to Equation (2) results 


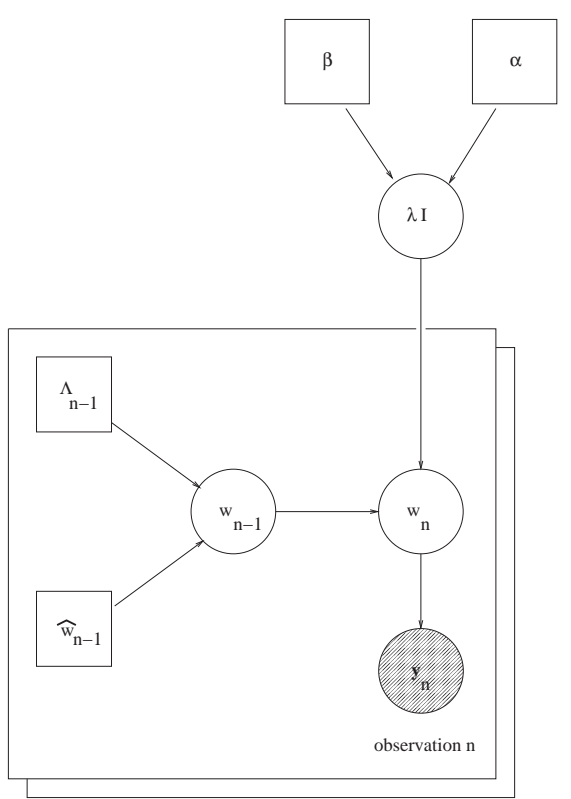

Fig. 1. We may illustrate adaptive inference as a directed acyclic graph. The coefficients of the classifier, $\boldsymbol{w}_{n}$, are assumed to be Gaussian, following a first order Markov process. The posterior at time $n-1, p\left(\boldsymbol{w}_{n-1} \mid \hat{\boldsymbol{w}}_{n-1}, \Lambda_{n-1}\right)$, together with the process noise specified by $\lambda \boldsymbol{I}$ determine the prior for $\boldsymbol{w}_{n}$. The hyper parameter $\lambda$ is given a flat Gamma prior specified by parameters $\alpha=0.01$ and $\beta=10^{-4}$. In order to make inference of $\lambda$ not too sensitive on the prior settings, we assume a constant adaption rate within a window of size 15 .

in the well known Kalman filter equations. Since we predict probabilities, we need to approximate a non-linearity, which we do with a variational method (e.g. [4] and [1]) to obtain a flexible but parametric posterior. The algorithm suggested in [12] performs inference sequentially. We first predict the cognitive state for an an EEG segment before we use the corresponding label to update the model parameters. A retrospective evaluation of the average communication bandwidth of the adaptive BCI was obtained using third order AR coefficients extracted from one second segments for both EEG channels. The labeled data were divided into two equally sized data sets. We use each dataset once to allow the algorithm to converge, before estimating the performance on the other dataset. Table II compares the bandwidth of truly adaptive BCI (column vkf) with the bandwidth of an equivalent static method (column vsi), which is the same model however assuming stationarity (practically we set $\lambda$ to $\infty$ ). McNemar's test reveals that even on a moderate number of trials we obtain statistically significant higher bit rates with the adaptive BCI. This suggests that long term use of BCI will clearly benefit from fully adaptive translation algorithms.

\section{Conclusion}

The results reported in this paper suggest probabilistic modifications of current BCI systems that lead to increased robustness and higher communication bandwidth. Our implementation of a fully probabilistic BCI suggests that the features we obtain from preprocessing are associated with uncertainty which must be taken into account if we want
TABLE II

COMMUNiCATION BANDWIDTH OF FULly ADAPTIVE VS. STATIC BCI

\begin{tabular}{|c|c|c|c|}
\hline cognitive tasks & vsi & vkf & $P_{\text {null }}$ \\
\hline aud. / navig. & $0.34 \mathrm{bit} / \mathrm{s}$ & $0.42 \mathrm{bit} / \mathrm{s}$ & 0.02 \\
\hline left / right & $0.22 \mathrm{bit} / \mathrm{s}$ & $0.27 \mathrm{bit} / \mathrm{s}$ & 0.04 \\
\hline
\end{tabular}

optimal bandwidths. A probabilistic line of thought also allows us to relax the stationarity assumption implied by current BCI technology and take temporal variations of task associated EEG dynamics into account. The resulting adaptive BCI might prevent, or at least postpone, failures that are observed in long term use of BCIs.

\section{ACKNOWLEDGEMENTS}

This work was supported by the BUPA foundation (Grant No. F46/399) and the Neuro-disability Trust (Grant No. NRT/99/1). We want to thank our colleagues Dr. W. Penny, Dr. I Johnsrude and Dr. A Owen for having been involved in the studies relating to this paper and the anonymous reviewers for useful suggestions.

\section{REFERENCES}

[1] H. Attias. Inferring parameters and structure of latent variable models by variational Bayes. In Proc. 15th Conf. on Uncertainty in $A I, 1999,1999$

[2] J. M. Bernardo and A. F. M. Smith. Bayesian Theory. Wiley, Chichester, 1994.

[3] N. Birbaumer, N. Ghanayim, T. Hinterberger, I. Iversen, B. Kotchoubey, A. Kubler, J. Perelmouter, E. Taub, and H. Flor. A spelling device for the paralysed. Nature, 398:297-298, 1999.

[4] M. I. Jordan, Z. Ghahramani, T. S. Jaakkola, and L. K. Saul. An introduction to variational methods for graphical models. In M. I. Jordan, editor, Learning in Graphical Models. MIT Press, Cambridge, MA, 1999.

[5] R. M. Neal. Bayesian Learning for Neural Networks. Springer, New York, 1996.

[6] B. Obermeier, C. Guger, C. Neuper, and G. Pfurtscheller. Hidden Markov models for online classification of single trial EEG. Pattern Recognition Letters, pages 1299-1309, 2001.

[7] W. Penny, S. Roberts, E. Curran, and M. Stokes. EEG-based communication: a pattern recognition approach. IEEE Trans. Rehab. Eng., pages 214-216, 2000.

[8] G. Pfurtscheller, D. Flotzinger, and J. Kalcher. Brain-Computer Interface - a new communication device for handicapped people. Journal of Microcomputer Applications, pages 293-299, 1993.

[9] B. D. Ripley. Pattern Recognition and Neural Networks. Cambridge University Press, Cambridge, 1996.

[10] J. J. K. Ó Ruanaidh and W. J. Fitzgerald. Numerical Bayesian Methods Applied to Signal Processing. Springer-Verlag, New York, 1995.

[11] P. Sykacek and S. Roberts. Bayesian time series classification. In T.G. Dietterich, S. Becker, and Z. Gharamani, editors, Advances in Neural Processing Systems 14, pages 937-944. MIT Press, 2002.

[12] P. Sykacek and S. Roberts. Adaptive classification by variational Kalman filtering. In S. Thrun, S. Becker, and K. Obermayer, editors, Advances in Neural Processing Systems 15. MIT Press, 2003.

[13] J. R. Wolpaw, N. Birbaumer, W. J. Heetderks, D. J. McFarland, P. H. Peckham, G. Schalk, E. Donchin, L. A. Quatrano, C. J. Robinson, and T. M. Vaughan. Brain-Computer Interface technology: a review of the first international meeting. IEEE Trans. Rehab. Eng., pages 164-173, 2000.

[14] J. R. Wolpaw, D. J. McFarland, D. J. Neat, and C. A. Forneris. An EEG-based Brain-Computer Interface for Cursor Control. Electroencephalography and Clinical Neurophysiology, 78:252259, 1991. 